UCRL-JC-124477

PREPRINT

\title{
Dispersive Wave Processing: A Model-Based Solution
}

\author{
J. V. Candy \\ D. C. Chambers
}

This paper was prepared for submittal to the

13th Annual Asilomar Conference on Signals, Systems and Computers

Pacific Grove, CA

November 3-6, 1996

October 30, 1996

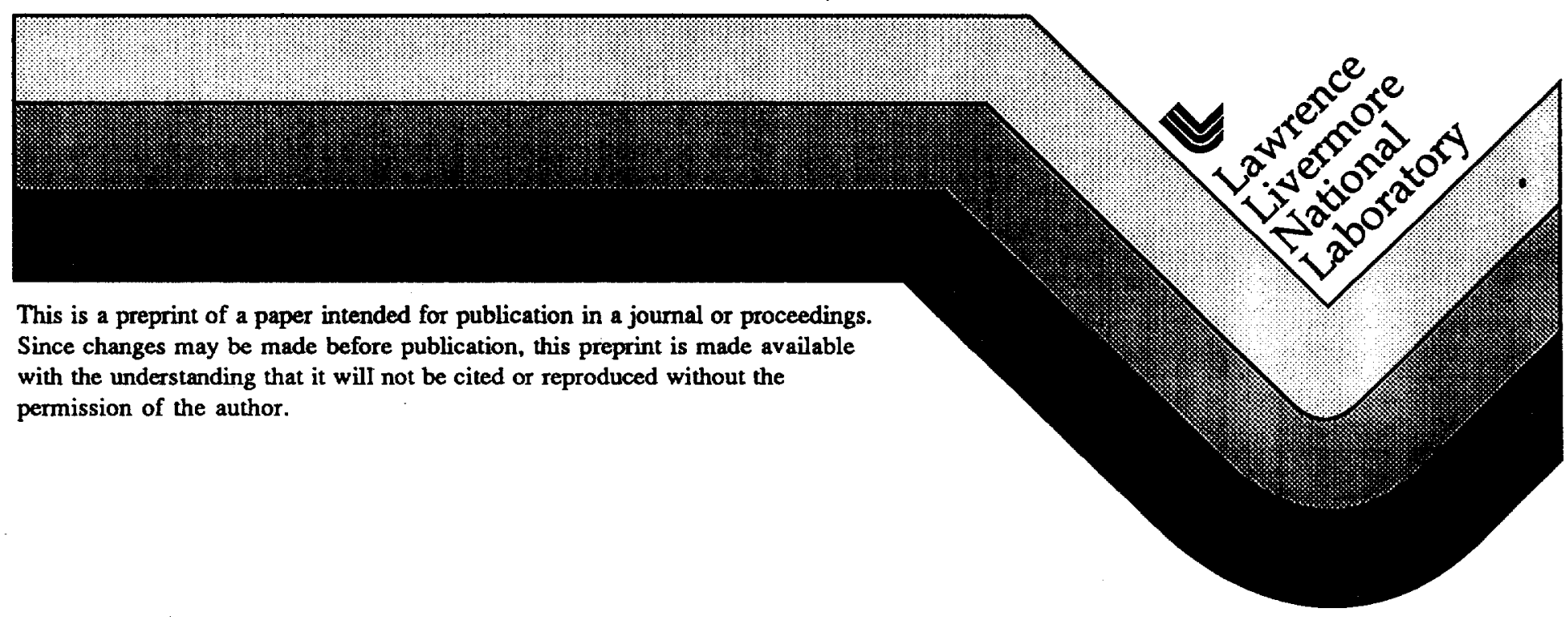




\section{DISCLAIMER}

This document was prepared as an account of work sponsored by an agency of the United States Government. Neither the United States Government nor the University of California nor any of their employees, makes any warranty, express or implied, or assumes any legal liability or responsibility for the accuracy, completeness, or usefulness of any information, apparatus, product, or process

disclosed, or represents that its use would not infringe privately owned rights. Reference herein to any specific commercial product, process, or service by trade name, trademark, manufacturer, or otherwise, does not necessarily constitute or imply its endorsement, recommendation, or favoring by the United States Government or the University of California. The views and opinions of authors expressed herein do not necessarily state or reflect those of the United States Government or the University of California, and shall not be used for advertising or product endorsement purposes. 


\title{
Dispersive Wave Processing: A Model-Based Solution
}

\author{
J. V. Candy D. C. Chambers \\ P.O. Box 808, L-437 \\ Lawrence Livermore National Laboratory \\ Livermore, CA 94550
}

\begin{abstract}
Wave propagation through various media represents a significant problem in many applications in acoustics and electromagnetics especially when the medium is dispersive. We pose a general dispersive wave propagation model that could easily represent many classes of dispersive waves and proceed to develop a model-based processor employing this underlying structure. The general solution to the model-based dispersive wave estimation problem is developed using the Bayesian maximum a-posteriori (MAP) approach which leads to the nonlinear extended Kalman filter (EKF) processor.
\end{abstract}

\section{Introduction}

Dispersive wave propagation through various media is a significant problem in many applications ranging from radar target identification where electromagnetic waves propagate through the atmosphere to discern the nature of a reflected pulse classifying an intruder as friend or foe, or in submarine detection and localization where the propagation of acoustic waves through the ever-changing dispersive ocean medium causes great concern when trying to detect the presence of a hostile target and track its movements. Therefore, there is a need to develop generic characterizations of dispersive waves which do not depend on the details of the physical system primarily because the required details such as governing equations for the system and their solutions may be imperfectly known. In this paper we will show that a model-based signal processing scheme applicable to any dispersive wave system can be developed from the basic properties of wave propagation in a dispersive medium.

Our approach will be to develop a state-space description of a dispersive wave measured by a sensor or array of sensors. For simplicity we restrict ourselves to one-dimensional waves, but the generalization to higher dimensions is straightforward. The wave pulse is assumed to be generated by an impulsive source at a known position and a known time. We consider the source pulse as a superposition of wave components of many frequencies. Since the system is dispersive, each component propagates at a different speed resulting in a spreading or dispersing of the pulse over space and time as it propagates. This spreading is described by the dispersion relation of the system which relates the frequency of each component to its wave number. We will show that a complete state-space representation of the wave can be formulated from the dispersion re- lation combined with an envelope or amplitude modulation function. The dispersion relation completely describes the propagation properties of the dispersive system, while the envelope is related to the initial conditions. Once specified, it is then possible to develop a generic model-based processing scheme for dispersive waves. The primary motivation for this approach follows the dispersive wave characterization developed in the text by Whitham [1]. This processor evolves directly from the modified plane wave, internal wave techniques developed using an approximation of the dispersion relation [2]. In contrast, the generic dispersive approach relies exclusively on the underlying envelope and dispersion relation to develop an optimal Bayesian processor. It is this model-based approach that we employ using a dynamic propagation model incorporated into an optimal estimation scheme to provide the necessary internal wave enhancement.

In section 1, we present the general dispersive wave representation and outline the corresponding modelbased processor (MBP) using the Bayesian maximum a posteriori (MAP) approach for the underlying wave estimation or equivalent signal enhancement problem. Next, employing this general solution, we apply it to the problem of internal wave estimation directly from an empirical dispersion relation in section 2 , while applying it to simulated data in section 3 .

\section{Model-Based Dispersive Processor}

In this section we develop the underlying dispersive wave model and cast it into state-space form. Once this is accomplished, a Bayesian maximum a posteriori solution is outlined and the resulting processor is shown to lead to the extended Kalman filter (EKF) solution [3]. The complete model-based solution is then specified in terms of the model and algorithm showing that intuitively the dispersion relation is the fundamental quantity that must be known a-priori.

\subsection{Dispersive State-Space Propagator}

First, we develop the state-space representation of a general dispersive wave system obtained from a simple physical characterization of a dispersive wave measured by a sensor or array of sensors. In constrast to the usual approach of classifying non-dispersive waves in terms of their inherent differential equations (hyperbolic, el- 
liptic, etc.), we use a solution rather than propagation equation for our dispersive prototype. Therefore, following Whitham [3], we define a generic dispersive wave as any system which admits solutions of the general form

$$
u(x, t)=\alpha(x, t) \sin [\theta(x, t)]
$$

where $u$ is the measured field and $\alpha(x, t), \theta(x, t)$ are the respective envelope or amplitude modulation and phase functions. The phase is assumed to be monotonic in $x$ and $t$, and the envelope is assumed to be slowly varying compared to the phase. The phase function describes the oscillatory character of a wave, while the slowly varying envelope allows modulation of the wave without destroying its wave-like character. The local values of wave number and frequency can be defined as

$$
\kappa(x, t) \equiv \frac{\partial \theta}{\partial x}, \quad \omega(x, t) \equiv-\frac{\partial \theta}{\partial t} .
$$

These functions are also assumed to be slowly varying and describe the frequency modulation of a dispersive wave train. By slowly varying we mean that we can approximate the phase function as

$$
\theta(x, t)=\kappa(x, t) x-\omega(x, t) t
$$

The combination of Eqs. 1 and 3 can be considered an asymptotic solution to some dispersive wave system. To complete the specification of a dispersive wave system, we define the dispersion relation, $\omega \equiv \omega(\kappa, x, t)$. This is generally an algebraic function of $\kappa(x, t)$ but can also depend on $x$ and $t$ separately to represent timevarying, nonuniform wave systems. Here we will write $\omega=\omega(\kappa)$ where the $x$ and $t$ dependence through the wave number function $\kappa(x, t)$ and any system nonuniformity is implied. This and the envelope are the only parts of the description which are unique to the particular type of wave system under investigation. The choice of dispersion relation enables the differentiation between acoustic radiation, electromagnetic radiation, ocean surface waves, internal gravity waves, or any other wave type. Thus, the dispersion relation is equivalent to the governing equations for a particular wave system [1]. Our only restriction on it in this paper is that it is independent of the envelope, $\alpha(x, t)$. This restricts our formulation to linear dispersive waves.

From Eqs. 2 and 3 it can be shown that the phase fronts of any wave travel at the phase speed defined by

$$
c_{p}(\kappa) \equiv \frac{\omega}{\kappa}
$$

while the points of constant wave number $\kappa$ travel at the group velocity defined by

$$
c_{g}(\kappa) \equiv \frac{\partial \omega}{\partial \kappa}
$$

These two speeds are not the same in general and are functions of wave number $\kappa$. The group velocity has the additional significance of being the energy propagation speed for the wave system, that is, the energy in the wave packet is carried at this velocity. As such it plays a central role in the state-space formulation of a general dispersive wave system.

Now consider the problem where an impulse occurs at time $t=0$ at the spatial origin, $x=0$. The impulse can be represented by the superposition of wave components with various wave numbers. A wave train is generated by the impulse and propagates aways from the origin. Each wave number component in the train propagates with group velocity given by Eq. 5 . If a sensor is placed at a distance $x$ away from the origin, then the local wave number, $\kappa(x, t)$, observed at time $t>0$ is related to $x$ by the group velocity,

$$
x=c_{g}(\kappa(x, t)) t
$$

This relation is simply a restatement of the definition of the group velocity as the speed at which a given wave number $\kappa(x, t)$ propagates in the wave train. It is the group velocity that plays the dominant role in dispersive wave propagation. Note also that the resulting wave train does not have constant wavelength, since the whole range of wave numbers is still present, that is, $\lambda(x, t)=\frac{2 \pi}{\kappa(x, t)}$.

The actual sensor measurement $u(t)$ at $x$ is given by combining Eqs. 1 and 3, that is,

$$
u(t)=\alpha(x, t ; \kappa) \sin [\kappa(t) x-\omega(\kappa) t]
$$

where we have suppressed the dependence of $\kappa$ on $x$ and allowed the envelope to be a function of $\kappa$. We will choose the wave number $\kappa(t)$ at $x$ as our state variable and develop a dynamical equation for its temporal evolution by differentiating $\mathrm{Eq}$. 6 using the chain rule

$$
\frac{d c_{g}(\kappa)}{d t}=\frac{d \kappa}{d t} \times \frac{d c_{g}(\kappa)}{d \kappa},
$$

to obtain

$$
0=\frac{d \kappa}{d t}\left[\frac{d c_{g}(\kappa)}{d \kappa} t\right]+c_{g}(\kappa)
$$

Now solving for $\frac{d \kappa}{d t}$ and substituting the expression for group velocity in terms of our original dispersion relation, we obtain

$$
\frac{d \kappa}{d t}=-\frac{1}{t}\left[\frac{d \omega(\kappa)}{d \kappa}\right]\left[\frac{d^{2} \omega(\kappa)}{d \kappa^{2}}\right]^{-1} ; \quad t>0
$$

which shows how the wave number evolves dynamically (temporally) as a function of the underlying dispersion, relation $\omega(\kappa)$. If we couple this expression back to the original dispersive wave solution, then we can have a general continuous-time, spatio-temporal, dispersive wave, state-space representation with state defined by $\kappa(t)$.

Suppose we sample this wave with an array of $L$ sensors oriented in the direction of propagation, that is, $x \rightarrow x_{\ell}, \ell=1, \cdots, L$, giving $L$ wave numbers and $L$ initial conditions. If the entire state-space is to be initialized at the same time, care must be taken to select the initialization time to be after the leading edge of the wave has passed through the entire array. Let $t_{o}$ be the time the leading edge passes the 
sensor $L$, the sensor farthest from the origin, then $x_{L}=c_{g}\left(\kappa_{L}\left(t_{o}\right)\right) t_{o}$, where $\kappa_{L}\left(t_{o}\right)$ is the wave number for the maximum group velocity. The initial conditions for the other sensors in the array are obtained by solving $x_{\ell}=c_{g}\left(\kappa_{\ell}\left(t_{0}\right)\right) t_{0}$ for each $\ell$. Thus, the "spatially" sampled, dispersive wave, state-space model is given by:

$$
\begin{aligned}
\frac{d \kappa_{\ell}}{d t} & =-\frac{1}{t}\left[\frac{d \omega\left(\kappa_{\ell}\right)}{d \kappa_{\ell}}\right]\left[\frac{d^{2} \omega\left(\kappa_{\ell}\right)}{d \kappa_{\ell}^{2}}\right]^{-1}, t \geq t_{o} ; \\
u_{\ell}(t) & =\alpha\left(t ; \kappa_{\ell}\right) \sin \left[\kappa_{\ell} x_{\ell}-\omega\left(\kappa_{\ell}\right) t\right] \\
& \kappa_{\ell}\left(t_{o}\right), \quad \ell=1, \cdots, L .
\end{aligned}
$$

We can further discretize this model temporally by sampling $t \rightarrow t_{k}$, and also by replacing the derivatives with their first difference approximations. Since we know that the dispersive medium in which the wave propagates is uncertain, we can also characterize uncertainties with statistical models, one of which is the well-known Gauss-Markov model [3]. Performing these operations we achieve our desired result, the discrete, spatio-temporal, dispersive wave state-space Gauss-Markov model:

$$
\begin{aligned}
\kappa_{\ell}\left(t_{k+1}\right) & =\kappa_{\ell}\left(t_{k}\right)-\frac{\Delta}{t}\left[\frac{d \omega}{d \kappa}\right]\left[\frac{d^{2} \omega}{d \kappa^{2}}\right]^{-1}+\Delta w_{\ell}\left(t_{k}\right) ; \\
u_{\ell}\left(t_{k}\right) & =\alpha \cdot\left(t_{k} ; \kappa_{\ell}\right) \sin \left[\kappa_{\ell}\left(t_{k}\right) x_{\ell}-\omega\left(\kappa_{\ell}\right) t_{k}\right]+v_{\ell}\left(t_{k}\right), \\
& \kappa_{\ell}\left(t_{o}\right) ; \quad \ell=1, \cdots, L
\end{aligned}
$$

where $w_{\ell}\left(t_{k}\right)$ and $v_{\ell}\left(t_{k}\right)$ are assumed zero mean, gaussian noise sources with respective covariances, $R_{w w}\left(t_{k}\right), R_{w w}\left(t_{k}\right)$. The general vector Gauss-Markov form can be found in Refr. 3 and is simply given by

$$
\begin{aligned}
& \kappa\left(t_{k+1}\right)=\mathbf{a}\left[\kappa, t_{k}\right]+\Delta \mathbf{w}\left(t_{k}\right), \\
& \mathbf{u}\left(t_{k}\right)=\mathbf{c}\left[\kappa, t_{k}\right]+\mathbf{v}\left(t_{k}\right),
\end{aligned}
$$

where $\mathbf{a}[\bullet], \mathbf{c}[\bullet]$ are the respective nonlinear vector system and measurement functions with the corresponding state and measurement covariances defined by: $\mathbf{P}\left(t_{k+1}\right)$ and $R_{u u}\left(t_{k}\right)$, with the system and measurement jacobians, $\mathbf{A}[\kappa] \equiv \frac{\partial \mathrm{a}}{\partial \kappa}$ and $\mathbf{C}[\kappa] \equiv \frac{\partial \mathrm{c}}{\partial \kappa}$. The subsequent development of our processor will rely on this statistical formulation for both simulation and estimation.

\subsection{Dispersive Model-Based Processor}

Next we outline the model-based processor (MBP) based on the vector representation of the wave numbers and wave-field, that is, we define the vectors, $\mathbf{u}\left(t_{k}\right) \equiv$ $\left[u_{1}\left(t_{k}\right), \cdots, u_{L}\left(t_{k}\right)\right]^{T}$ and $\kappa \equiv\left[\kappa_{1}\left(t_{k}\right), \cdots, \kappa_{L}\left(t_{k}\right)\right]^{T}$. Once the wave is characterized by the underlying Gauss-Markov representation, then dispersive wave estimation problem can be specified by

GIVEN the approximate Gauss-Markov model (above) characterized by the dispersive wave state-space model and a set of noisy measurements, $\left\{\mathbf{u}\left(t_{k}\right)\right\}, F I N D$ the best (minimum error variance) estimate of the wave, that is, find $\hat{\mathbf{u}}\left(t_{k}\right)$.

The minimum variance solution to this problem can be obtained by the maximizing a posteriori density, leading to the so-called $M A P$ equation,

$$
\left.\frac{\partial}{\partial \kappa} \ln \operatorname{Pr}\left(\kappa\left(t_{k+1}\right) \mid U_{k+1}\right)\right|_{\kappa=\hat{\kappa}_{M A P}}=0
$$

Differentiating the posterior density and noting that $\hat{\kappa}\left(t_{k+1 \mid k}\right)$ and $\epsilon\left(t_{k}\right)$ are both functions of the data set, $U_{k}$, we obtain that $\hat{\kappa}_{M A P}\left(t_{k+1}\right)=\hat{\kappa}\left(t_{k+1 \mid k+1}\right)$ as (see Refr. 3, pp. 80-81 for more details)

$$
\hat{\kappa}\left(t_{k+1 \mid k+1}\right)=\hat{\kappa}\left(t_{k+1 \mid k}\right)+\mathbf{K}\left(t_{k+1}\right) \epsilon\left(t_{k+1}\right),
$$

where this expression is the corrected estimate (below) and shown in the algorithm of Table 6.63 of [3]. Thus, the model-based solution to this wave enhancement problem can be achieved using the nonlinear extended Kalman filter (EKF) algorithm which is given (simply) as:

Prediction:

$\hat{\kappa}_{\ell}\left(t_{k+1 \mid k}\right)=\hat{\kappa}_{\ell}\left(t_{k \mid k}\right)-\frac{\Delta}{t}\left[\frac{d \omega}{d \kappa}\right]\left[\frac{d^{2} \omega}{d \kappa^{2}}\right]^{-1}, \ell=1, \cdots, L$

Innovation:

$$
\begin{array}{ll}
\epsilon\left(t_{k}\right) & =\mathbf{u}\left(t_{k}\right)-\hat{\mathbf{u}}\left(t_{k+1 \mid k}\right) \\
\hat{u}_{\ell}\left(t_{k+1 \mid k}\right) & =\alpha\left(t_{k} ; \hat{\kappa}_{\ell}\left(t_{k+1 \mid k}\right)\right) \sin \left[\hat{\kappa}_{\ell}\left(t_{k+1 \mid k}\right) x_{\ell}-\omega^{\prime}\left(\hat{\kappa}_{\ell}\right) t_{k}\right] \\
& \ell=1, \cdots, L
\end{array}
$$

Correction:

$$
\hat{\kappa}\left(t_{k+1 \mid k+1}\right)=\hat{\kappa}\left(t_{k+1 \mid k}\right)+\mathbf{K}\left(t_{k}\right) \epsilon\left(t_{k}\right)
$$

Gain:

$$
\mathbf{K}\left(t_{k}\right)=\tilde{\mathbf{P}}\left(t_{k+1 \mid k}\right) \mathbf{C}^{T}\left(t_{k}\right) \mathbf{R}_{c \epsilon}^{-1}\left(t_{k}\right)
$$

Here the predicted and corrected covariances are given in Table 6.63. From the Table we see that in order to construct the optimal dispersive wave modelbased processor, we must not only specify the required initial conditions, but also the respective system and measurement jacobians: $\frac{d a|\bullet|}{d \kappa}$ and $\frac{d c|\vartheta|}{d \kappa}$. For our general solution, we see that

$$
\begin{aligned}
& a\left[\kappa_{\ell}, t_{k}\right]=\kappa_{\ell}\left(t_{k}\right)-\frac{\Delta}{t}\left[\frac{d \omega}{d \kappa}\right]\left[\frac{d^{2} \omega}{d \kappa^{2}}\right]^{-1}, \\
& c\left[\kappa_{\ell}, t_{k}\right]=\alpha\left(t_{k} ; \kappa_{\ell}\right) \sin \left[\kappa_{\ell}\left(t_{k}\right) x_{\ell}-\omega\left(\kappa_{\ell}\right) t_{k}\right], \ell=1 \ldots L . L .
\end{aligned}
$$


The jacobians then follow easily as

$$
\begin{aligned}
A\left[\kappa_{\ell}\left(t_{k}\right), t_{k}\right] & =1-\frac{\Delta}{t}\left(1-\frac{\left[\frac{d \omega}{d \kappa}\right]\left[\frac{d^{3} \omega}{d \kappa^{3}}\right]}{\left[\frac{d^{2} k^{2}}{d \kappa^{2}}\right]^{2}}\right), \ell=1, \cdots, L ; \\
C\left[\kappa_{\ell}\left(t_{k}\right), t_{k}\right] & =\frac{d \alpha_{\ell}\left(t_{k}\right)}{d \kappa_{\ell}} \sin \left[\kappa_{\ell}\left(t_{k}\right) x_{\ell}-\omega(\kappa) t_{k}\right] \\
& +\alpha_{\ell}\left(t_{k}\right) \cos \left[\kappa_{\ell}\left(t_{k}\right) x_{\ell}-\omega t_{k}\right]\left(x_{\ell}-\frac{d \omega}{d \kappa} t_{k}\right)
\end{aligned}
$$

This completes the section on dispersive wave estimation, next we consider the application of this processor for internal waves.

\section{Internal Wave Processor}

When operating in a stratified environment with relatively sharp density gradients any excitation that disturbs the pycnocline (density profile) will generate internal waves [4].

To apply our processor to the internal wave enhancement problem, we first recall the original dispersive wave system of Eqns. 1 and 3 and apply this structure to the internal wave dynamics where $u$ represents the measured velocity field and $\alpha(x, t)$ and $\theta(x, t)$ are the respective envelope and phase to be specified by the internal wave structure. We define the internal wave dispersion relation by

$$
\omega \equiv \omega_{o}(\kappa)+\kappa(x, t) \nu .
$$

where we have included the additive velocity term to account for the effects of a doppler shift created by the ambient current. That is, in the original formulation we have replaced the position variable by $x \rightarrow x-\nu t$ which leads to the above equation. For $\omega_{0}(\kappa)$, we use a dispersion model based on some empirical results, the Barber approximation [5], for internal wave dispersion and group velocity. Thus, we have

$$
\omega_{\circ}(\kappa)=\frac{C_{o} \kappa(t)}{1+\frac{C_{o}}{N_{o}} \kappa(t)}
$$

with $C_{0}$ is the initial phase velocity and $N_{0}$ is the maximum of the Brunt-Väisälä frequency profile. It is also possible to derive the following approximation to the amplitude modulation function as

$$
\alpha\left(t_{k}\right)=\frac{A}{\sqrt{t}}\left[c_{p}(\kappa)\right]^{3 / 2} \sin \left[\omega\left(\kappa, t_{k}\right) T_{w}\right]
$$

where $A$ is a constant amplitude governing the overall envelope gain, $T_{w}$ is a temporal window width and $c_{p}(\kappa)$ is the phase speed defined below.

We use the simulator [6] to synthesize internal wave dynamics corresponding to an internal wave field experiment performed in Loch Linnhe, Scotland in 1994 $[7,8]$. The simulation (shown in Figures 1a) was performed based on the $S N R$ defined by: $S N R:=\sigma^{2} / R_{v v}$, where $\sigma$ is the energy in the true image (scaled to unit variance) and $R_{v v}$ is the measurement noise variance extracted from experimental data. The spatiotemporal velocity field includes the entire range of 361 temporal samples at $\Delta t=5 \mathrm{sec}$ representing a propagation time of approximately $1 / 2$ hour and spatially we assume a line array of $\mathbf{3 0}$ sensors (enough to illustrate the wave structure) spaced at $\triangle x=4 m$ representing an aperture of 120 meters. For this internal wave simulation, we choose a peak Brunt-Väisälä frequency of $N_{0}=0.137 r / \mathrm{sec}$ and a long wave $(\kappa=0)$ phase speed of $C_{o}=0.34 \mathrm{~m} / \mathrm{s}$ with the ambient current (doppler shift) of $\nu=-0.1 \mathrm{~cm} / \mathrm{sec}$. The data was contaminated with additive gaussian noise at a $-23 d B S N R$. Here we observe the effect of additive gaussian noise in obscurring (visually) the internal wave dynamics of the synthesized velocity field. We see the noisy internal wave spatio-temporal signals synthesized, while the enhanced wave estimates are shown in $1 \mathrm{~b}$. The results appear quite good, however, they must be analyzed from the statistical perspective to actually assess the overall performance of the processor. With this information in hand, we specify the required dispersive wave statespace model as:

$$
\begin{aligned}
& \kappa_{\ell}\left(t_{k+1}\right)=\kappa_{\ell}\left(t_{k}\right)+\frac{\Delta t_{k}}{t_{k}} \frac{N_{0}}{2} \frac{1+\frac{1}{c_{g o}\left(\kappa_{\ell}\right)}}{\sqrt{C_{o} c_{g_{o}}\left(\kappa_{\ell}\right)}}, \quad t_{k} \geq t_{o i} \\
& u_{\ell}\left(t_{k}\right)=\alpha_{\ell}\left(t_{k}\right) \sin \left[\kappa_{\ell}\left(t_{k}\right) x_{\ell}-\left(\omega_{o}\left(\kappa_{\ell}\right)+\kappa_{\ell}\left(t_{k}\right) \nu\right) t_{k}\right] \\
& \ell=1, \cdots, L \text {. }
\end{aligned}
$$

Thus, the MAP estimator can now be constructed using the formulation of the previous section and substituting the internal wave model and above noted jacobians.

We used SSPACK_PC; a model-based signal processing toolbox available in MATLAB to design the processor $[9,10]$. The model-based wave enhancer is able to extract the internal wave signatures quite effectively even though the embedded dispersive wave model is just an approximation to the actual wave dynamics. We show the spatio-temporal interpretation (and display) of the noisy and enhanced signals in Figure 1. To confirm the processor performance that we observed visually, we perform individual whiteness tests on each of the temporal sensor outputs. We use the corresponding sensor innovation indicating the difference between the noisy and predicted measurement along with the bounds predicted by the processor. Here $95 \%$ of the innovations should lie within the bounds indicating a reasonable processor. Performing these tests on all of the sensor innovation outputs reveals that each individually satisfied these statistical tests indicating a zero mean/white innovations and a near optimal processor. Thus, these simulations show that the GaussMarkov formulation enables us to capture various uncertainties of internal waves as well as its associated statistics in a completely consistent framework.

To further assess the feasibility of this approach, we ran the enhancer on other synthetic data sets at $0 d B,-10 d B$, and $-13 d B$ with similar results, that is, the model-based approach enabled a near-optimal Bayesian solution with all sensor innovation sequences statistically testing as zero-mean/white. We are currently applying this technique to carefully controlled experimental measurements and the preliminary results have led us to continue to pursue this approach. 

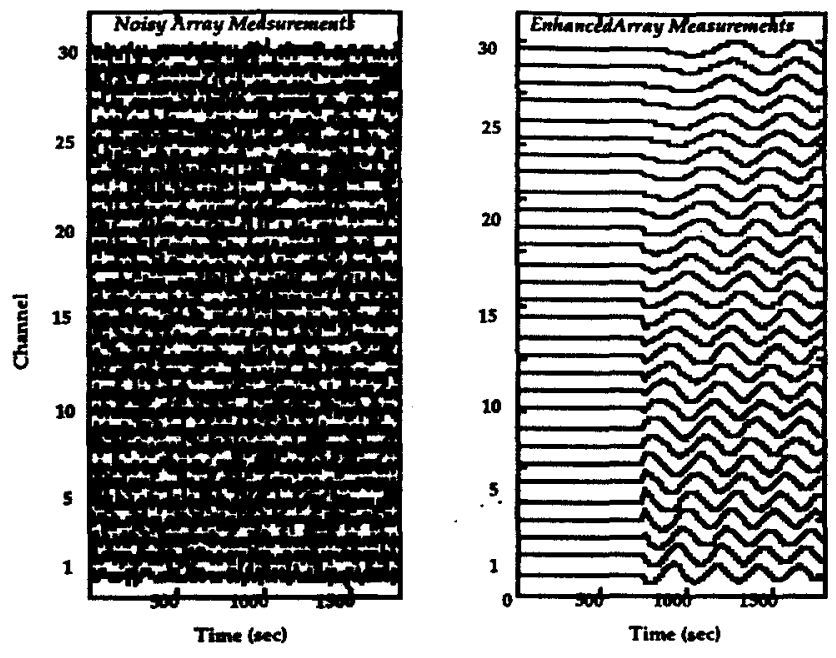

Figure 1: Equivalent Model-Based Internal Wave Spatio-Temporal Enhancement: (a) Synthesized wave (-23 dB SNR). (b) Enhanced internal wave.

\section{Summary}

In this paper we have developed a generic dispersive wave processor. Starting with a general solution to the propagation of waves in a dispersive medium, we developed the approximate (due to nonlinear systems) maximum a-posterior (MAP) solution using a Bayesian formulation of the wave field enhancement problem. The results are significant in that all that is required is the envelope or equivalently amplitude modulation function and dispersion relation to completely specify the underlying wave system. It is in fact the particular dispersion relation that enables the differentiation between acoustic and electromagnetic radiation, ocean surface waves and internal gravity waves or seismic waves or any wave system for that matter. The generality of this result enables the specification of a particular wave system by its underlying envelope and dispersion and then applying the algorithm to obtain the MAP solution.

\section{References}

[1] G. B. Whitham, Linear and Nonlinear Waves, New York: Wiley, 1974.

[2] J. V. Candy and D. H. Chambers. "Internal wave processing: a model-based approach." IEEE J. Oceanic Engr., Vol. 21, (1), 37-52, 1996.

[3] J. V. Candy, Signal Processing: The Model-Based Approach. New York:McGraw-Hill, 1986.

[4] J. R. Apel, Principles of Ocean Physics, New York:Academic Press, 1987.
[5] B. C. Barber, "On the dispersion relation for trapped internal waves," J. Fluid Mechanics., 252, 31-49, 1993.

[6] M. Milder, Internal Waves Radiated by a Moving Source: Analytic Simulation, RDA Report, RDATR-2702-007, 1974.

[7] D. D. Mantrom, "Loch Linnhe '94: Test Operations Description and On-Site Analysis US Activities," LLNL Report.,UCRL-ID-119197, 1994.

[8] H. F. Robey and D. L. Ravizza, "Loch Linnhe Experiment 1994: Background Stratification and Shear Measurements Part 1: Profile Summary and Dispersion Relations", LLNL Report, UCRL-ID$119352,1994$.

[9] J. V. Candy and P. M. Candy, "SSPACK_PC: A model-based signal processing package on personal computers," DSP Applic., 2,(3), 33-42, 1993.

[10] MATLAB. Boston: The Math Works Inc., 1993.

[11] J. V. Candy and D. H. Chambers. "Model-based enhancement of internal wave images." to appear, Special Issue on Imaging in the Ocean, IEEE $J$. Oceanic Engr., Jan. 1997.

\section{Acknowledgements}

We would like to acknowledge the motivation and support of Dr. R. Twogood, Program Leader LLNL Imaging \& Detection Program and Mr. James Brase, Project Leader for the Radar Ocean Imaging Project. This work was performed under the auspices of the Department of Energy by the Lawrence Livermore $\mathrm{Na}$ tional Laboratory under contract W-7405-Eng-48. 


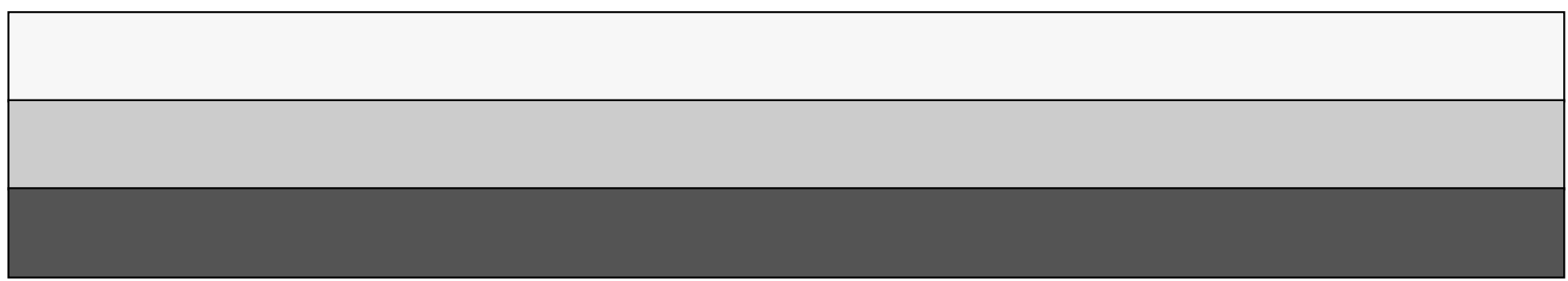

